\title{
Myocardial Perfusion in Rheumatoid Arthritis Patients: Associations with Traditional Risk Factors and Novel Biomarkers
}

\author{
Miguel Bernardes, ${ }^{1,2}$ Tiago S. Vieira, ${ }^{3}$ Maria João Martins, ${ }^{4,5}$ Raquel Lucas, ${ }^{6,7}$ Lúcia Costa, ${ }^{1}$ \\ Jorge G. Pereira, ${ }^{3}$ Francisco Ventura, ${ }^{8}$ and Elisabete Martins ${ }^{2,4,9}$ \\ ${ }^{1}$ Department of Rheumatology, São João Hospital Center, Porto, Portugal \\ ${ }^{2}$ Department of Medicine, Faculty of Medicine, University of Porto, Alameda Prof. Hernâni Monteiro, 4200-319 Porto, Portugal \\ ${ }^{3}$ Department of Nuclear Medicine, São João Hospital Center, Porto, Portugal \\ ${ }^{4}$ i3s-Instituto de Investigação e Inovação em Saúde, Universidade do Porto, Porto, Portugal \\ ${ }^{5}$ Department of Biomedicine, Unit of Biochemistry, Faculty of Medicine, University of Porto, Porto, Portugal \\ ${ }^{6}$ Department of Clinical Epidemiology, Predictive Medicine and Public Health, Faculty of Medicine, University of Porto, \\ Porto, Portugal \\ ${ }^{7}$ EPI Unit-Institute of Public Health, University of Porto, Porto, Portugal \\ ${ }^{8}$ Faculty of Medicine, University of Porto, Porto, Portugal \\ ${ }^{9}$ Department of Cardiology, São João Hospital Center, Porto, Portugal
}

Correspondence should be addressed to Miguel Bernardes; mbernardes09@gmail.com

Received 26 December 2016; Accepted 2 April 2017; Published 3 May 2017

Academic Editor: Lorenzo Cavagna

Copyright (C) 2017 Miguel Bernardes et al. This is an open access article distributed under the Creative Commons Attribution License, which permits unrestricted use, distribution, and reproduction in any medium, provided the original work is properly cited.

Introduction. Cardiovascular (CV) diseases are a major cause of death in rheumatoid arthritis (RA) patients. Novel biomarkers [B-type natriuretic peptide (BNP); osteoprotegerin (OPG)/receptor activator of nuclear factor-kappa B ligand (RANKL) ratio; and dickkopf-1 (DKK-1)] have been used in CV risk assessment. We analysed, in established RA patients, the presence of silent myocardial ischemia and its association with clinical variables, BNP, and bone and atheroma biomarkers. Methods. From a singlecenter tertiary referral hospital, RA patients asymptomatic for CV disease were submitted to myocardial perfusion scintigraphy (MPS) under adenosine stress and biomarkers measurements. Logistic regression was used to estimate crude odds ratios (OR) and $95 \%$ confidence intervals (CI). Results. In 189 patients, perfusion defects were frequent (25\%) and associated with BNP $\geq$ $100 \mathrm{pg} / \mathrm{mL}(\mathrm{OR}=5.68$; 95\% CI: 2.038-15.830), fourth $\log$ OPG/RANKL ratio quartile (OR = 2.88; 95\% CI: 1.091-7.622), and DKK$1 \geq 133 \mathrm{pmol} / \mathrm{L}(\mathrm{OR}=2.69 ; 95 \% \mathrm{CI}$ : 1.058-6.840). Similar associations were confirmed in those with C-reactive protein $>$ or $\leq$ $3 \mathrm{mg} / \mathrm{L}$. No relationship was found with the majority of traditional CV factors nor with disease variables. Conclusions. Our results corroborated the hypothesis that MPS could reveal subclinical CV dysfunction, supported the utility of BNP measurements as a screening tool, and put in perspective the potential usefulness of complementary approaches in CV risk assessment in RA patients.

\section{Introduction}

Cardiovascular diseases (CVD) are a major cause of death in rheumatoid arthritis (RA) patients [1] and clinical events occur approximately 10 years earlier than in the general population [2]. The pooled relative risk of CVD is near 1.5 [3] and cardiac risk is similar to that of type 2 diabetes mellitus (T2DM), especially in RA patients with long disease duration [4].

The CV risk increases in ongoing disease but is already higher than in non-RA subjects at the time of RA diagnosis [5]. The risk seems to be less associated with the traditional 
(arterial hypertension, diabetes, dyslipidemia, and obesity) or other known CV risk factors (hyperuricemia, hyperhomocysteinemia, and vitamin D deficiency) and more with the disease itself (inflammatory and autoimmune mechanisms) and therapeutic related variables [6]. The relationships with and between each risk factor are complex and have been progressively unraveled in recent years [6].

Considering the increased risk, the EUropean League Against Rheumatism (EULAR) recommends annual CV risk assessment in RA patients (or every 2 to 3 years, if low $\mathrm{CV}$ risk and inactive disease occur) [7], with calculation of a modified SCORE index based on traditional CV risk factors, multiplied by 1.5 in the presence of 2 out of 3 RA factors: disease duration $>10$ years, certain extra-articular manifestations, or positivity for rheumatoid factor (RF) or anti-cyclic citrullinated peptides (anti-CCP) antibodies.

However, there are uncertainties about the effectiveness of this method in individual CV risk appraisal [5]. In line, several authors have pointed out the need for complementary approaches, including CV imaging techniques and the quantification of newly available biomarkers [8-10].

Myocardial perfusion scintigraphy (MPS) is an imaging method robustly validated in CV risk assessment in several categories of patients [11].

On the other hand, in recent years, new biomarkers that can reflect the interplay between inflammation, atherosclerosis, and plaque instability phenomena have been tested on clinical grounds and some seem promising in $\mathrm{CV}$ risk estimation. Serum B-type natriuretic peptides have been clearly associated with CV death and, in RA patients, with $\mathrm{CV}$ and general mortality [9]. Less data and conflicting results still exist in what regards the association between another group of mediators, such as osteoprotegerin (OPG) [10], the receptor activator of nuclear factor-kappa B ligand (RANKL) [12], or dickkopf-1 (DKK-1) [13], and the development of CVD in RA.

In this study we included MPS in our risk stratification protocol of patients with established RA. We aimed to evaluate the prevalence of myocardial perfusion defects and to relate the MPS results with various clinical features, including disease characteristics, therapy, traditional CV risk factors, and serum biomarkers [B-type natriuretic peptide (BNP), OPG, RANKL, DKK-1, and sclerostin].

\section{Patients and Methods}

2.1. Rheumatoid Arthritis Patients. During a six-month period, patients with RA, classified according to the 1987 American College of Rheumatology criteria [14] and asymptomatic for cardiac disease (angina or heart failure signs and symptoms), were prospectively included in the study protocol. In this time-frame, a total of 216 RA patients were eligible, although only 189 accepted to perform MPS.

The exclusion criteria were history of previous ischemic heart disease, presence of angina, heart failure, or Q waves on basal electrocardiogram (ECG).

Through a rheumatology appointment, all subjects underwent clinical assessment using the Portuguese version of the Stanford Health Assessment Questionnaire (HAQ) and the Disease Activity Score (DAS28) four variables (4v) [15], the most commonly used tools in daily clinical practice to determine the RA disease activity. All data regarding past and ongoing prescribed medications, including NSAIDs and corticosteroids exposure time, were obtained from medical records review. Corticosteroids daily dose was the one at the study inclusion time.

Hypertension, diabetes, and dyslipidemia were defined by self-reporting and contemporary use of compatible medications and/or clinical data assessment in previous medical registries. Office blood pressure (>140/90 $\mathrm{mmHg}$ ), lipid profile (total cholesterol $>200 \mathrm{mg} / \mathrm{dL}$ ), and fasting plasma glucose $(<126 \mathrm{mg} / \mathrm{dL})$ were categorized according to the European guidelines. The smoking status (past and current) was registered and the body mass index (BMI) calculated.

2.2. Laboratory Measurements. Between 08:00 and 10:00 hours in the morning, a fasting blood sample was collected to evaluate the lipid profile [high-density lipoprotein (HDL) cholesterol, low-density lipoprotein (LDL) cholesterol, total cholesterol (TC), triglycerides, lipoprotein (a), apolipoprotein $\mathrm{Al}$ (ApoA1), and apolipoprotein B (ApoB)], as well as glucose and insulin, C-reactive protein (CRP), erythrocyte sedimentation rate (ESR), RF and anti-CCP antibodies, homocysteine, uric acid, $25(\mathrm{OH})$ vitamin $\mathrm{D} 3$, parathyroid hormone, osteocalcin, BNP, OPG, RANKL, DKK-1, and sclerostin levels. Serum samples were stored at $-70^{\circ} \mathrm{C}$ for $\mathrm{OPG}$, RANKL, DKK-1, and sclerostin measurements by ELISA. All these specimens were measured in duplicate, according to the manufacturer's instructions and then averaged. OPG and DKK-1 were determined using kits from BIOMEDICA immunoassays (Vienna, Austria); the intra-assay coefficients of variation $(\mathrm{aCV})$ were $\leq 3 \%$ for both; the inter-aCV was $\leq 3 \%$ for DKK- 1 and between 3 and $5 \%$ for OPG. RANKL was determined using a kit from CUSABIO (Wuhan, China); the intra-aCV was $<8 \%$ and the inter-aCV was $<10 \%$. Sclerostin was determined using a kit from TECO (Sissach, Switzerland); the intra- and inter-aCVs were $3.8-8.0 \%$ and $3.3-9.0 \%$, respectively.

2.3. Myocardial Perfusion Scintigraphy. For minimization of exposure to radiation, all patients underwent a routine procedure used in our institution of adenosine-stress single photon emission-computed tomography (SPECT) myocardial perfusion imaging, in a one-day protocol, with the use of stress-first imaging protocol. Images at rest were only acquired if any perfusion defects were detected after the stress phase. This protocol implies the injection of 10 (at stress) to 30 (at rest) $\mathrm{mCi}$ doses of TC-99m tetrofosmin, adenosine infused at a rate of $140 \mu \mathrm{g} / \mathrm{kg} / \mathrm{min}$ over $6 \mathrm{~min}$, and radiopharmaceutical administration at $3 \mathrm{~min}$ of adenosine perfusion.

SPECT imaging was acquired in a Gamma Camera Infinia Hawkeye, GE Healthcare, 15-30 min after radiopharmaceutical administration.

The SPECT images were interpreted in a blinded fashion, by two experienced physicians, consecutively during the 
study protocol, interspersed with other MPS in patients without RA. Bearing in mind the female predominance [16, 17] attenuation correction (AC) was applied on a case by case basis, depending on patient BMI $\left(\geq 30 \mathrm{~kg} / \mathrm{m}^{2}\right)$ and breast volume, using an integrated computed tomography (CT) scanner. A 3 min low-dose CT scan (140 keV, $2.5 \mathrm{mAs}$ ) was acquired at the end of stress and rest acquisitions.

Myocardial perfusion was assessed through expert visual analysis in a 17 left ventricle (LV) segmentation model. QGS/QPS ${ }^{\circledR}$ software was used to quantify the extension and severity of perfusion defects, wall motion and wall thickening scores, LV end-diastolic and end-systolic volumes (EDV and ESV), and ejection fraction (EF).

Myocardial perfusion was considered abnormal only if the summed stress score (SSS) was $\geq 4$. Perfusion defects were classified as mild (SSS $\geq 4$ and $\leq 8$ ), moderate (SSS $>8$ and $\leq 13$ ), or severe (SSS $>13$ ) and myocardial ischemia was considered significant if the summed difference score (SDS) with respect to MPS at rest was $\geq 4$ [18].

2.4. Ethics. Informed consent was obtained from each patient. The study protocol conforms to the ethical guidelines of the 1975 Declaration of Helsinki as reflected in a priori approval (on March 13, 2008) by the institution's human research committee.

2.5. Follow-Up Data. All patients were followed during a fiveyear period. Death and CV events, including the onset of cardiac symptoms, were registered.

2.6. Statistical Analyses. Qualitative data are described as absolute counts and proportions and quantitative data as mean (standard deviation). CRP, ESR, and OPG/RANKL ratio were log-transformed to obtain symmetrical distributions. The magnitude of the associations between clinical and laboratory variables and the presence of defects in MPS were assessed using logistic regression models in order to estimate crude odds ratios (OR) and their 95\% confidence intervals (CI). All analyses were two-sided and $p$ values < 0.05 were considered statistically significant. Since this study was oriented by a priori defined hypotheses no adjustments for multiple comparisons were conducted, as supported by previous literature [19]. Statistical analyses were performed using STATA software V.11 (Statacorp, College Station, Texas, USA).

\section{Results}

3.1. Patients Characteristics. One hundred and eighty-nine patients with RA were included, with a mean age of $53 \pm$ 12 years, the majority $(n=153,81 \%)$ females. Disease duration, measured from the date of diagnosis, was $14 \pm$ 10 years. The majority of patients presented erosive disease $(n=187,99 \%)$ and were seropositive for RF $(n=115$, $61 \%)$ and anti-CCP $(n=152,80 \%)$ antibodies. The mean DAS28 $(4 \mathrm{v})$ and HAQ were $4.266 \pm 1.324$ and $1.275 \pm$ 0.708 , respectively. DAS28 remission was present in $18(10 \%)$ patients. Extra-articular manifestations were present in 67
(35.4\%) patients: Sjögren syndrome (20\%), rheumatoid nodes $(16 \%)$, interstitial lung disease (3\%), AA amyloidosis (3\%), scleritis (3\%), and rheumatoid vasculitis (2\%).

Clinical and laboratory variables are shown in Table 1.

3.2. Relation of Myocardial Perfusion Scintigraphy Findings with Clinical and Laboratory Parameters. On basal ECG, 184 (97\%) patients were in sinus rhythm and 5 (3\%) had left bundle branch blockage (LBBB).

MPS showed myocardial perfusion defects (SSS $\geq 4$ ) in $47(25 \%)$ patients, mild defects in $35(18.5 \%)$, moderate in 10 (5.3\%), and severe in $2(1 \%)$. The defects had a mean SDS of 4.1 \pm 3 and stress extension of $2.5 \pm 3.6 \%$ and were reversible at rest in 31 (16\%) cases. LV ejection fraction (LVEF) was inferior to $45 \%$ in $9(4.76 \%)$ patients with a mean value of $63.7 \pm 9.8 \%$.

The perfusion defects were either unique (25/47, 53\%) or multiple $(22 / 47,47 \%)$, with no preferential distribution at different coronary territories.

Figure 1 illustrates an abnormal MPS in female RA patient without any cardiac symptom.

On logistic regression analysis, no relationship was found between the presence of myocardial perfusion defects and patient age, gender, RA disease or therapy related features, smoking habits, history of dyslipidemia, diabetes, or hypertension, neither with determinations, at inclusion protocol, of serum levels of lipids, glucose, or insulin. MPS findings were associated with BMI $(\mathrm{OR}=1.120 ; p=0.002)$, therapy with ARAs $(\mathrm{OR}=2.471 ; p=0.046)$, diuretics $(\mathrm{OR}=2.195$; $p=0.045)$, and oral antidiabetic $(\mathrm{OR}=3.031 ; p=0.033)$, as well as with circulating levels of $\mathrm{BNP} \geq 100 \mathrm{pg} / \mathrm{mL}$ (OR $=5.680 ; p=0.001), \mathrm{DKK}-1 \geq 133 \mathrm{pmol} / \mathrm{L}(\mathrm{OR}=2.690$; $p=0.038$ ), and fourth log OPG/RANKL ratio quartile (OR $=2.884 ; p=0.033)$ (Table 2$)$.

In a subgroup analysis of those who presented CRP higher than $3 \mathrm{mg} / \mathrm{L}$ (upper limit of normality for our laboratory), we found the same associations with similar magnitudes, except for ARAs and diuretics use. MPS findings were associated with BMI $(\mathrm{OR}=1.174 ; p=0.001)$, oral antidiabetics $(\mathrm{OR}=$ 3.840; $p=0.017)$, levels of $\mathrm{BNP} \geq 100 \mathrm{pg} / \mathrm{mL}(\mathrm{OR}=5.610$; $p=0.004), \mathrm{DKK}-1 \geq 133 \mathrm{pmol} / \mathrm{L}(\mathrm{OR}=3.142 ; p=0.056)$, and fourth $\log$ OPG/RANKL ratio quartile $(\mathrm{OR}=2.410 ; p=$ 0.001).

3.3. Follow-Up Data: Cardiovascular Events. During a period of 5 years, 9 patients deceased, 2 from CV causes: two sudden cardiac deaths, one of them after an acute myocardial infarction (MI) (confirmed by autopsy). In these two male patients, LVEF was inferior to $45 \%$ and both presented perfusion defects on MPS.

Apart from the CV deaths, three other patients developed CV events. A male patient, with $\mathrm{LBBB}$ without perfusion defects and with normal LVEF, had MI four years after MPS evaluation. A female patient, with a mild perfusion defect and normal LVEF, developed angina and was submitted to percutaneous stent implantation in the anterior descendent coronary artery. Another female patient, without perfusion defects and with normal LVEF, had a stroke four years after MPS evaluation. All these 5 patients with CV events were 
TABLE 1: Clinical and laboratory variables of the rheumatoid arthritis sample enrolled in the study.

\begin{tabular}{|c|c|}
\hline Characteristic & Value \\
\hline \multicolumn{2}{|l|}{ Patient related } \\
\hline Female, $n(\%)$ & $153(81.0)$ \\
\hline Postmenopausal, $n(\%)$ & $59(31.2)$ \\
\hline Time since menopause, mean (SD), years & $7.330(8.275)$ \\
\hline Age, mean (SD), years & $53.340(11.502)$ \\
\hline BMI, mean (SD), $\mathrm{kg} / \mathrm{m}^{2}$ & $27.150(4.768)$ \\
\hline Ever smokers, $n(\%)$ & $24(12.7)$ \\
\hline Hypertension, $n(\%)$ & $39(20.6)$ \\
\hline Diabetes mellitus, $n(\%)$ & $14(7.4)$ \\
\hline Dyslipidemia, $n(\%)$ & $23(12.2)$ \\
\hline \multicolumn{2}{|l|}{ Disease related } \\
\hline Disease duration, mean (SD), years & $13.72(9.955)$ \\
\hline RF positivity, $n(\%)$ & $115(60.847)$ \\
\hline Anti-CCP antibodies positivity, $n(\%)$ & $152(80.423)$ \\
\hline DAS28 (4V), mean (SD) & $4.266(1.324)$ \\
\hline Remission $(\mathrm{DAS} 28(4 \mathrm{v})<2.6)$ & $18(9.5)$ \\
\hline Low $(2.6<$ DAS $28(4 \mathrm{v}) \leq 3.2)$ & $27(14.3)$ \\
\hline Moderate $(3.2<$ DAS28 $(4 \mathrm{~V}) \leq 5.1)$ & $95(50.3)$ \\
\hline High $($ DAS28 $(4 v)>5.1)$ & $49(25.9)$ \\
\hline ESR, mean (SD), mm (first hour) & $27.100(20.603)$ \\
\hline CRP, mean (SD), mg/L & $11.573(20.045)$ \\
\hline Global health on VAS, mean (SD) & $44.190(17.794)$ \\
\hline HAQ score, mean (SD) & $1.275(0.708)$ \\
\hline \multicolumn{2}{|l|}{$R A$ related treatment } \\
\hline Corticosteroids, $n(\%)$ & $155(82.0)$ \\
\hline Daily dose in prednisolone equivalents, mean (SD), mg & $5.139(3.977)$ \\
\hline Time under corticosteroids, mean (SD), years & $11.429(8.981)$ \\
\hline NSAIDs, $n(\%)$ & $142(75.1)$ \\
\hline DMARDS, $n(\%)$ & $160(84.7)$ \\
\hline Cumulative, mean (SD) & $2.534(1.416)$ \\
\hline Time under DMARDs, mean (SD), months & $104.037(87.714)$ \\
\hline Methotrexate, $n(\%)$ & $130(68.8)$ \\
\hline Leflunomide, $n(\%)$ & $40(21.1)$ \\
\hline Biologic DMARDs, $n(\%)$ & $97(51.3)$ \\
\hline Time under biologics, mean (SD), months & $60.258(30.080)$ \\
\hline Anti-TNF $\alpha$ blockers, $n(\%)$ & $83(43.9)$ \\
\hline Non-anti-TNF $\alpha$ blockers, $n(\%)$ & $14(7.4)$ \\
\hline Bisphosphonates, $n(\%)$ & $69(36.5)$ \\
\hline \multicolumn{2}{|l|}{ Cardiovascular related treatment } \\
\hline Antiaggregants, $n(\%)$ & $22(11.6)$ \\
\hline ACEIs, $n(\%)$ & $24(12.7)$ \\
\hline ARAs, $n(\%)$ & $24(12.7)$ \\
\hline CCAs, $n(\%)$ & $18(9.5)$ \\
\hline BBs, $n(\%)$ & $15(7.9)$ \\
\hline Diuretics, $n(\%)$ & $37(19.6)$ \\
\hline Warfarin, $n(\%)$ & $5(2.6)$ \\
\hline Statins, $n(\%)$ & $45(23.8)$ \\
\hline Oral antidiabetic agents, $n(\%)$ & $17(9.0)$ \\
\hline Insulin, $n(\%)$ & $5(2.6)$ \\
\hline
\end{tabular}

ACEIs, angiotensin converter enzyme inhibitors; ARAs, angiotensin receptor antagonists; BBs, beta-blockers; BMI, body mass index; CCAs, calcium channel antagonists; CCP, cyclic citrullinated peptides; CRP, c-reactive protein; DAS, disease activity score; DMARDS, disease-modifying antirheumatic dugs; ESR, erythrocyte sedimentation rate; HAQ, health assessment questionnaire; NSAIDs, nonsteroidal anti-inflammatory drugs; RF, rheumatoid factor; SD, standard deviation; TNF $\alpha$, tumour necrosis factor alpha; VAS, visual analogic scale. 

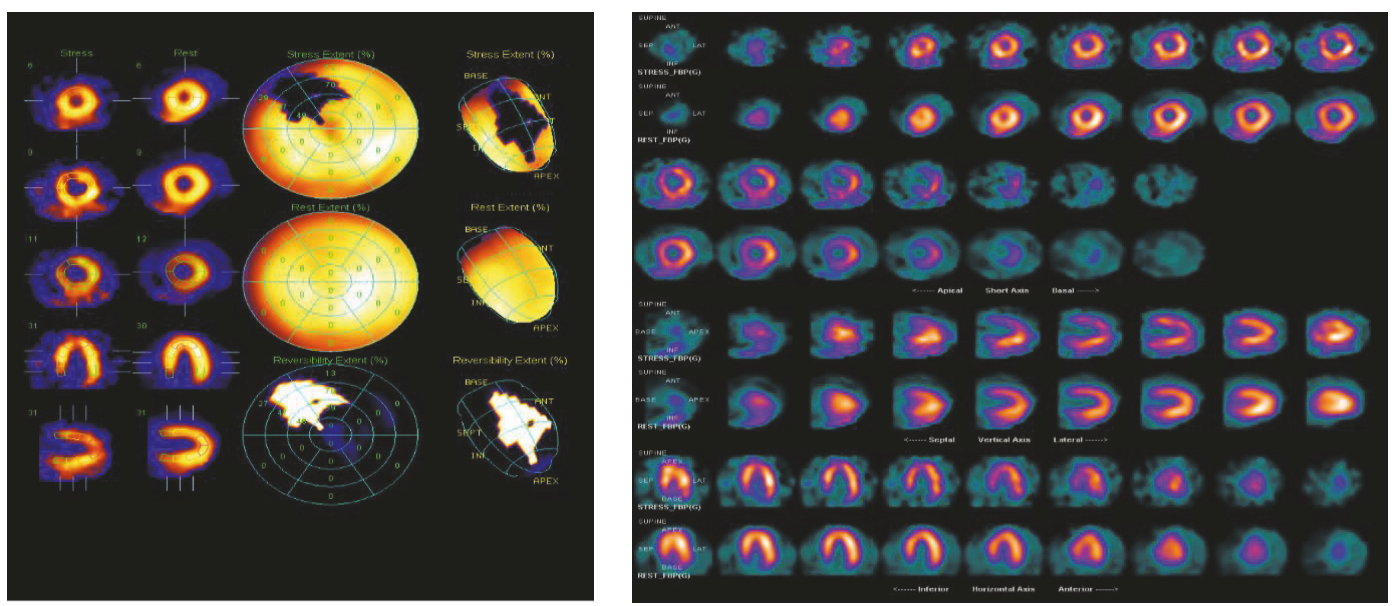

FIGURE 1: Moderate and reversible anteroseptal perfusion defect compatible with ischemia in the left anterior descending coronary artery territory in an asymptomatic female RA patient.

seropositive for RF and anti-CCP antibodies, were nonobese, and had low to moderate disease activity by DAS28 and a normal BNP value at baseline and at least one traditional CV risk factor under treatment.

\section{Discussion}

The major finding of this study comprised the description of myocardial perfusion scintigraphy findings in a large RA patient sample, characterized by a long disease duration, with moderate activity, and with no apparent cardiac symptoms. The relationship of MPS results with several clinical variables, including traditional CV risk factors and novel biomarkers of atherosclerosis, was also evaluated.

$\mathrm{RA}$ is characterized by earlier atherosclerosis and CV events in comparison with the general population [2, 20]. Overall, the pooled relative risk is 1.68 and 1.87 for myocardial infarction and congestive heart failure (HF), respectively [3]. Myocardial ischemia is often asymptomatic and clinical detection is frequently made at an advanced stage. HF is a major cause of morbidity and mortality in RA, accounting for about $20 \%$ of mortality in patients with RA [21]. The risk of HF has been associated with RF positivity, ESR, severe extraarticular involvement, and steroids use [22].

As most CV events are of cardiac origin, it seems appropriate to consider the integration of cardiac imaging in primary prevention strategies, at least in the highest risk patients. However we have few clinical tools able to a priori identify these patients and, until now, few studies have assessed the presence of silent ischemia in large RA patients populations [23].

MPS is a robustly validated risk stratification method in different populations, and a normal MPS is associated with $\leq 1 \% /$ year combined mortality and nonfatal infarction rate [24]. It is a noninvasive functional test that depends not only on the presence of epicardial coronary stenosis (macrovascular disease) but also on the endothelial function, including in the small vessels territories (microvascular disease), which may be early affected during disease development and can be modulated by the inflammatory state.

It is important to note that the nature of the perfusion defects can have an ischemic/atherosclerotic etiology but could also result from other causes like cardiac amyloidosis [8] or interstitial fibrosis. The clinical impact of discriminating the different causes of perfusion defects, for instance, by cardiac magnetic resonance is still unknown.

No relationship was found between the MPS results and the classic CV risk factors. The lack of associations could be explained by the well-known complex interplay between inflammation and therapy that could alter the link between risk factors and atherosclerosis manifestations, as already pointed by other authors [25].

The fact that in our patients we did not find relations between MPS results and the traditional risk factors can thus be explained by the fact that the usual relationships were changed by the inflammatory RA disease activity and/or by medications such as with nonbiologic $(85 \%)$ or biological (51\%) DMARDs.

ESR and CRP levels are related to disease activity and inflammation; seropositivity also increases $\mathrm{CV}$ risk in RA patients [26]. On the other hand, high CRP levels are related to an increased intima media thickness [27] and baseline values also predict accelerated brachial arterial wall changes in patients with recent-onset RA [28].

In our study, the presence of perfusion defects on MPS was not related to systemic inflammatory markers (CRP and ESR), nor with the presence of RF and anti-CCP antibodies or DAS28 score.

The inflammatory state could have changed over time, especially under therapy, and, later in disease progression, it could be difficult to relate a unique plasma level (mean CRP value of $11.57 \pm 20.0 \mathrm{mg} / \mathrm{L}$ in our patients) with the extension of a long lasting process of cardiac damage.

B-type natriuretic peptides (BNP and NT-proBNP) are independent predictors of $\mathrm{CV}$ morbidity and of all causes of mortality including in RA patients. 
TABLE 2: Crude odds ratios for the associations between rheumatoid arthritis patients' characteristics and the presence of myocardial perfusion defects obtained by logistic regression analysis.

\begin{tabular}{|c|c|c|c|c|}
\hline & \multicolumn{4}{|c|}{$95 \%$ CI for OR } \\
\hline & OR & Lower & Upper & $p$ value \\
\hline Male sex (versus female) & 1.009 & 0.436 & 2.334 & 0.984 \\
\hline Age (per year) & 1.000 & 0.971 & 1.029 & 0.989 \\
\hline Disease duration (per year) & 1.013 & 0.981 & 1.047 & 0.435 \\
\hline$B M I\left(\right.$ per $\left.\mathrm{kg} / \mathrm{m}^{2}\right)$ & 1.120 & 1.044 & 1.202 & 0.002 \\
\hline Ever smoker (versus never smoker) & 1.008 & 0.375 & 2.711 & 0.987 \\
\hline Smoking exposure (per year) & 1.002 & 0.918 & 1.093 & 0.971 \\
\hline Time under biologics & 1.002 & 0.993 & 1.010 & 0.729 \\
\hline DAS28 (4v) (per unit) & 0.913 & 0.710 & 1.176 & 0.482 \\
\hline HAQ (per unit) & 0.930 & 0.582 & 1.485 & 0.760 \\
\hline $\log$ CRP (per unit) & 1.129 & 0.872 & 1.464 & 0.357 \\
\hline log ESR (per unit) & 0.896 & 0.562 & 1.428 & 0.644 \\
\hline \multicolumn{5}{|l|}{$B N P(p g / m L)$} \\
\hline$<30$ & Reference & Reference & Reference & Reference \\
\hline$\geq 30$ and $<100$ & 1.635 & 0.780 & 3.428 & 0.193 \\
\hline$\geq 100$ & 5.680 & 2.038 & 15.830 & 0.001 \\
\hline \multicolumn{5}{|l|}{ Insulin resistance } \\
\hline$<1$ & Reference & Reference & Reference & Reference \\
\hline$\geq 1$ and $<1.5$ & 2.260 & 0.816 & 6.263 & 0.117 \\
\hline$\geq 1.5$ and $<2.5$ & 1.361 & 0.500 & 3.707 & 0.546 \\
\hline$\geq 2.5$ & 1.005 & 0.322 & 3.135 & 0.994 \\
\hline TC/HDL ratio (per unit) & 0.987 & 0.704 & 1.386 & 0.941 \\
\hline LDL/HDL ratio (per unit) & 0.980 & 0.643 & 1.495 & 0.927 \\
\hline ApoB/ApoA1 ratio (per unit) & 0.400 & 0.066 & 2.424 & 0.319 \\
\hline $\mathrm{TC}($ per mg/dL) & 0.935 & 0.368 & 2.377 & 0.888 \\
\hline LDL (per mg/dL) & 0.952 & 0.295 & 3.070 & 0.934 \\
\hline HDL (per mg/dL) & 1.005 & 0.103 & 9.801 & 0.996 \\
\hline TGs (per mg/dL) & 1.338 & 0.774 & 2.314 & 0.297 \\
\hline ApoA1 (per mg/dL) & 1.004 & 0.992 & 1.016 & 0.508 \\
\hline ApoB (per mg/dL) & 0.995 & 0.980 & 1.010 & 0.526 \\
\hline Lp(a) (mg/dL) (per unit) & 0.985 & 0.969 & 1.002 & 0.076 \\
\hline Uric acid (per mg/L) & 1.010 & 0.989 & 1.032 & 0.359 \\
\hline Homocysteine (per $\mu \mathrm{mol} / \mathrm{L}$ ) & 1.013 & 0.931 & 1.103 & 0.757 \\
\hline 25(OH) vitamin D3 (per ng/mL) & 1.023 & 0.998 & 1.048 & 0.077 \\
\hline Osteocalcin (per ng/mL) & 1.010 & 0.968 & 1.054 & 0.651 \\
\hline$D K K-1$ (pmol/L) (per unit) & 1.010 & 1.002 & 1.017 & 0.010 \\
\hline$<60$ & Reference & Reference & Reference & Reference \\
\hline$\geq 60$ and $<90$ & 0.778 & 0.263 & 2.297 & 0.649 \\
\hline$\geq 90$ and $<133$ & 1.657 & 0.630 & 4.355 & 0.306 \\
\hline$\geq 133$ & 2.690 & 1.058 & 6.840 & 0.038 \\
\hline Sclerostin (per ng/mL) & 2.071 & 0.063 & 68.436 & 0.683 \\
\hline $\log O P G / R A N K L$ (per unit) & 1.667 & 1.106 & 2.513 & 0.015 \\
\hline First quartile & Reference & Reference & Reference & Reference \\
\hline Second quartile & 1.357 & 0.481 & 3.828 & 0.564 \\
\hline Third quartile & 1.537 & 0.553 & 4.268 & 0.410 \\
\hline Fourth quartile & 2.884 & 1.091 & 7.622 & 0.033 \\
\hline Osteoprotegerin (per pmol/L) & 1.161 & 0.987 & 1.366 & 0.072 \\
\hline RANKL (per pmol/L) & 0.999 & 0.999 & 1.000 & 0.111 \\
\hline Prednisolone (mg a day) (per unit) & 1.066 & 0.984 & 1.154 & 0.119 \\
\hline
\end{tabular}


TABLE 2: Continued.

\begin{tabular}{|c|c|c|c|c|}
\hline & & & & \\
\hline & OR & Lower & Upper & $p$ value \\
\hline Corticosteroid therapy duration (years) (per unit) & 1.010 & 0.974 & 1.047 & 0.588 \\
\hline Methotrexate (mg once a week) (per unit) & 0.992 & 0.952 & 1.034 & 0.713 \\
\hline NSAIDs use (current versus not current) & 0.823 & 0.391 & 1.737 & 0.610 \\
\hline Bisphosphonates use (ever versus never) & 0.671 & 0.330 & 1.365 & 0.271 \\
\hline ACEIs use (current versus not current) & 1.615 & 0.643 & 4.060 & 0.308 \\
\hline ARAs use (current versus not current) & 2.471 & 1.015 & 6.018 & 0.046 \\
\hline CCAs use (current versus not current) & 1.585 & 0.560 & 4.490 & 0.386 \\
\hline BBs use (current versus not current) & 1.108 & 0.335 & 3.660 & 0.867 \\
\hline Diuretics use (current versus not current) & 2.195 & 1.018 & 4.732 & 0.045 \\
\hline Antidyslipidemic therapy (current versus not current) & 0.934 & 0.430 & 2.029 & 0.863 \\
\hline Insulin therapy (current versus not current) & 2.059 & 0.333 & 12.715 & 0.437 \\
\hline Oral antidiabetic therapy (current versus not current) & 3.031 & 1.096 & 8.382 & 0.033 \\
\hline Diabetes mellitus & 1.759 & 0.559 & 5.539 & 0.334 \\
\hline Arterial hypertension & 1.460 & 0.671 & 3.180 & 0.340 \\
\hline
\end{tabular}

ACEIs, angiotensin converter enzyme inhibitors; Apo, apolipoprotein; ARAs, angiotensin receptor antagonists; BBs, beta-blockers; BMI, body mass index; BNP, brain natriuretic peptide; CCAs, calcium channel antagonists; CI, confidence interval; CRP, c-reactive protein; ESR, erythrocyte sedimentation rate; DAS, disease activity score; DKK-1, dickkopf-1; HAQ, health assessment questionnaire; HDL, high-density lipoprotein; LDL, low-density lipoprotein; Lp(a), lipoprotein (a); NSAIDs, nonsteroidal anti-inflammatory drugs; OPG, osteoprotegerin; OR, odds ratio; RANKL, receptor activator of nuclear factor-kappa B ligand; TC, total cholesterol; TGs, triglycerides.

In early inflammatory polyarthritis, NT-proBNP has been associated with HAQ score and CRP [29] and this fact may be indicative of the link between joint and cardiac (myocardial and/or vascular) structural damage induced by chronic inflammatory diseases.

In our RA patients, with a longer disease duration, BNP was poorly correlated with HAQ (Spearman correlation; $r=$ $0.189, p=0.007$ ), but levels superior to $100 \mathrm{pg} / \mathrm{mL}$ were strongly associated with the presence of perfusion defects on MPS independently of LV EF.

This result corroborates the hypothesis that MPS could reveal subclinical cardiac dysfunction and supports the utility of BNP measurement as a screening tool in the assessment of $\mathrm{CV}$ risk in RA patients. This hypothesis needs to be corroborated with further studies.

OPG and RANKL are bone-controlling cytokines of mineral metabolism but also participate in the process of atherogenesis, calcification, and plaque rupture at the vascular walls [30].

In vascular atherogenic remodeling process, OPG could be increased due to a secondary compensatory mechanism activation [31], and higher OPG serum levels have been associated with coronary atherosclerosis [32], silent myocardial ischemia, increased CV mortality [33], myocardial infarction, and heart failure prognosis [34].

By contrast, RANKL promotes calcification at the vascular wall and it has been mostly linked with the risk of plaque instability and rupture [35].

In our study, we did not find associations between myocardial perfusion defects with either RANKL or OPG isolated levels, but rather with the highest quartile of the distribution of the OPG/RANKL ratio. This elevated ratio may be due to the presence of an ongoing vascular atherosclerosis (high OPG) in a still stable and low-risk plaques (low RANKL) in patients with abnormal MPS.

DKK-1 and sclerostin, two Wnt pathway inhibitors, are also involved in the atherosclerosis process. DKK-1 is linked not only with the effects of TNF $\alpha$ on joints in RA [36] but also with platelet-mediated endothelial cell activation [13], endothelial dysfunction in T2DM [37], vascular calcification [38], and premature myocardial infarction [39]. DKK1 serum concentration has already been correlated with the presence of coronary artery calcification and atherosclerosis [40], and its measurement has been proposed as a simple test that might be useful in CV risk stratification. Our results corroborate this last hypothesis since we found a significant association of DKK-1 levels with the presence of myocardial perfusion defects.

The occurrence of few number of $\mathrm{CV}$ events in our patients precludes statistical analysis for predictor variables but significant reductions of cardiovascular events have been reported with the use of biological compared with conventional DMARDs in RA patients [41-43].

\section{Study Limitations}

The major limitation of this study is related with AC in MPS which was not performed in all examinations and this might have contributed to overestimating the prevalence of perfusion defects in some patients; however, AC might also introduce false-positive perfusion defects particularly among the female population.

On the other hand, there was no coronary anatomy control that can guarantee the atherosclerotic nature of all perfusion defects nor the presence of balanced ischemia. 
The low representativeness of male and early RA patients precludes the extrapolation of obtained results to both genders and to patients with less disease duration.

\section{Conclusions}

A significant proportion of asymptomatic RA patients presented myocardial perfusion abnormalities in MPS. The association with BNP, OPG/RANKL ratio, and DKK-1 levels put in perspective the use of complementary approaches, including serum biomarkers quantification in CV risk stratification. Our results corroborated the hypothesis that MPS could reveal subclinical cardiac dysfunction and supported the utility of BNP measurement as a screening tool in CV risk assessment of RA patients.

More studies are needed to definitely evaluate the utility of different strategies (imaging, biomarkers) in the clinical setting, particularly in the era of increasing availability of RA biological therapies.

\section{Conflicts of Interest}

The authors declare that there are no conflicts of interest.

\section{Acknowledgments}

The authors wish to acknowledge Conceição Gonçalves (M.S.), Alexandra Bernardo (M.D.), and Sofia Pimenta (M.D.). This work was supported by the Associação Nacional de Reumatologia, Portugal.

\section{References}

[1] H. R. Kramer and J. T. Giles, "Cardiovascular disease risk in rheumatoid arthritis: progress, debate, and opportunity," Arthritis Care \& Research, vol. 63, no. 4, pp. 484-499, 2011.

[2] J. Lindhardsen, O. Ahlehoff, G. H. Gislason et al., "The risk of myocardial infarction in rheumatoid arthritis and diabetes mellitus: a Danish nationwide cohort study," Annals of the Rheumatic Diseases, vol. 70, no. 6, pp. 929-934, 2011.

[3] J. A. Avina-Zubieta, J. Thomas, M. Sadatsafavi, A. J. Lehman, and D. Lacaille, "Risk of incident cardiovascular events in patients with rheumatoid arthritis: a meta-analysis of observational studies," Annals of the Rheumatic Diseases, vol. 71, no. 9, pp. 1524-1529, 2012.

[4] M. J. L. Peters, V. P. Van Halm, A. E. Voskuyl et al., "Does rheumatoid arthritis equal diabetes mellitus as an independent risk factor for cardiovascular disease? A prospective study," Arthritis Care and Research, vol. 61, no. 11, pp. 1571-1579, 2009.

[5] A. M. Kerola, M. J. Kauppi, T. Kerola, and T. V. M. Nieminen, "How early in the course of rheumatoid arthritis does, the excess cardiovascular risk appear?" Annals of the Rheumatic Diseases, vol. 71, no. 10, pp. 1606-1615, 2012.

[6] E. Bartoloni, Y. Shoenfeld, and R. Gerli, "Inflammatory and autoimmune mechanisms in the induction of atherosclerotic damage in systemic rheumatic diseases: two faces of the same coin," Arthritis Care and Research, vol. 63, no. 2, pp. 178-183, 2011.
[7] M. J. L. Peters, D. P. M. Symmons, D. McCarey et al., "EULAR evidence-based recommendations for cardiovascular risk management in patients with rheumatoid arthritis and other forms of inflammatory arthritis," Annals of the Rheumatic Diseases, vol. 69, no. 2, pp. 325-331, 2010.

[8] S. Mavrogeni, T. Dimitroulas, P. P. Sfikakis, and G. D. Kitas, "Heart involvement in rheumatoid arthritis: multimodality imaging and the emerging role of cardiac magnetic resonance," Seminars in Arthritis and Rheumatism, vol. 43, no. 3, pp. 314324, 2013.

[9] S. A. Provan, K. Angel, A. G. Semb, D. Atar, and T. K. Kvien, "NT-proBNP predicts mortality in patients with rheumatoid arthritis: results from 10-year follow-up of the EURIDISS study," Annals of the Rheumatic Diseases, vol. 69, no. 11, pp. 1946-1950, 2010.

[10] R. Mogelvang, S. Haahr-Pedersen, M. Bjerre et al., "Osteoprotegerin improves risk detection by traditional cardiovascular risk factors and hsCRP," Heart, vol. 99, no. 2, pp. 106-110, 2013.

[11] T. Sharir, G. Germano, X. Kang et al., "Prediction of myocardial infarction versus cardiac death by gated myocardial perfusion SPECT: risk stratification by the amount of stress-induced ischemia and the poststress ejection fraction," Journal of Nuclear Medicine, vol. 42, no. 6, pp. 831-837, 2001.

[12] W. Lieb, P. Gona, M. G. Larson et al., "Biomarkers of the osteoprotegerin pathway: clinical correlates, subclinical disease, incident cardiovascular disease, and mortality," Arteriosclerosis, Thrombosis, and Vascular Biology, vol. 30, no. 9, pp. 1849-1854, 2010.

[13] T. Ueland, K. Otterdal, T. Lekva et al., "Dickkopf-1 enhances inflammatory interaction between platelets and endothelial cells and shows increased expression in atherosclerosis," Arteriosclerosis, Thrombosis, and Vascular Biology, vol. 29, no. 8, pp. 1228-1234, 2009.

[14] F. C. Arnett, S. M. Edworthy, D. A. Bloch et al., "The american rheumatism association 1987 revised criteria for the classification of rheumatoid arthritis," Arthritis \& Rheumatism, vol. 31, no. 3, pp. 315-324, 1988.

[15] M. L. L. Prevoo, M. A. Van 'T Hof, H. H. Kuper, M. A. Van Leeuwen, L. B. A. Van De Putte, and P. L. C. M. Van Riel, "Modified disease activity scores that include twenty-eight-joint counts: development and validation in a prospective longitudinal study of patients with rheumatoid arthritis," Arthritis and Rheumatism, vol. 38, no. 1, pp. 44-48, 1995.

[16] A. Wolak, P. J. Slomka, M. B. Fish, S. Lorenzo, D. S. Berman, and G. Germano, "Quantitative diagnostic performance of myocardial perfusion SPECT with attenuation correction in women," Journal of Nuclear Medicine, vol. 49, no. 6, pp. 915-922, 2008.

[17] J. M. Links, L. C. Becker, and F. Anstett, "Clinical significance of apical thinning after attenuation correction," Journal of Nuclear Cardiology, vol. 11, no. 1, pp. 26-31, 2004.

[18] R. Hachamovitch, D. S. Berman, L. J. Shaw et al., "Incremental prognostic value of myocardial perfusion single photon emission computed tomography for the prediction of cardiac death: differential stratification for risk of cardiac death and myocardial infarction," Circulation, vol. 97, no. 6, pp. 535-543, 1998.

[19] K. J. Rothman, "No adjustments are needed for multiple comparisons," Epidemiology, vol. 1, no. 1, pp. 43-46, 1990. 
[20] M. A. Gonzalez-Gay, C. Gonzalez-Juanatey, J. A. MirandaFilloy, C. Garcia-Porrua, J. Llorca, and J. Martin, "Cardiovascular disease in rheumatoid arthritis," Biomedicine and Pharmacotherapy, vol. 60, no. 10, pp. 673-677, 2006.

[21] P. J. Nicola, C. S. Crowson, H. Maradit-Kremers et al., "Contribution of congestive heart failure and ischemic heart disease to excess mortality in rheumatoid arthritis," Arthritis and Rheumatism, vol. 54, no. 1, pp. 60-67, 2006.

[22] E. Myasoedova, C. S. Crowson, P. J. Nicola et al., "The influence of rheumatoid arthritis disease characteristics on heart failure," Journal of Rheumatology, vol. 38, no. 8, pp. 1601-1606, 2011.

[23] S. Momose, "Detection of myocardial lesions by dipyridamole thallium-201 scintigraphy in patients with rheumatoid arthritis," Ryumachi, vol. 35, no. 3, pp. 559-565, 1995.

[24] G. Romero-Farina, J. Candell-Riera, S. Aguadé-Bruix et al., "Warranty periods for normal myocardial perfusion stress SPECT," Journal of Nuclear Cardiology, vol. 22, no. 1, pp. 44-54, 2015.

[25] M. A. González-Gay and C. González-Juanatey, "Inflammation and lipid profile in rheumatoid arthritis: bridging an apparent paradox," Annals of the Rheumatic Diseases, vol. 73, no. 7, pp. 1281-1284, 2014.

[26] E. Myasoedova, C. S. Crowson, H. M. Kremers et al., "Lipid paradox in rheumatoid arthritis: the impact of serum lipid measures and systemic inflammation on the risk of cardiovascular disease," Annals of the Rheumatic Diseases, vol. 70, no. 3, pp. 482-487, 2011.

[27] M. A. Gonzalez-Gay, C. Gonzalez-Juanatey, A. Piñeiro, C. Garcia-Porrua, A. Testa, and J. Llorca, "High-grade C-reactive protein elevation correlates with accelerated atherogenesis in patients with rheumatoid arthritis," Journal of Rheumatology, vol. 32, no. 7, pp. 1219-1223, 2005.

[28] S. Hannawi, T. H. Marwick, and R. Thomas, "Inflammation predicts accelerated brachial arterial wall changes in patients with recent-onset rheumatoid arthritis," Arthritis Research and Therapy, vol. 11, no. 2, article R51, 2009.

[29] H. Mirjafari, P. Welsh, S. M. M. Verstappen et al., "N-terminal pro-brain-type natriuretic peptide (NT-pro-BNP) and mortality risk in early inflammatory polyarthritis: results from the Norfolk Arthritis Registry (NOAR)," Annals of the Rheumatic Diseases, vol. 73, no. 4, pp. 684-690, 2014.

[30] A. Callegari, M. L. Coons, J. L. Ricks, M. E. Rosenfeld, and M. Scatena, "Increased calcification in osteoprotegerin-deficient smooth muscle cells: dependence on receptor activator of NF$\kappa \mathrm{B}$ ligand and interleukin 6," Journal of Vascular Research, vol. 51, no. 2, pp. 118-131, 2014.

[31] S. Morony, Y. Tintut, Z. Zhang et al., "Osteoprotegerin inhibits vascular calcification without affecting atherosclerosis in ldlr ${ }^{(-/-)}$mice," Circulation, vol. 117, no. 3, pp. 411-420, 2008.

[32] C. Pérez de Ciriza, M. Moreno, P. Restituto et al., "Circulating osteoprotegerin is increased in the metabolic syndrome and associates with subclinical atherosclerosis and coronary arterial calcification," Clinical Biochemistry, vol. 47, no. 18, pp. 272-278, 2014.

[33] M. Bjerre, J. Hilden, J. Kastrup et al., "Osteoprotegerin independently predicts mortality in patients with stable coronary artery disease: The CLARICOR trial," Scandinavian Journal of Clinical and Laboratory Investigation, vol. 74, no. 8, pp. 657-664, 2014.

[34] R. Røysland, S. Masson, T. Omland et al., "Prognostic value of osteoprotegerin in chronic heart failure: the GISSI-HF trial," American Heart Journal, vol. 160, no. 2, pp. 286-293, 2010.
[35] W. J. Sandberg, A. Yndestad, E. Øie et al., "Enhanced T-cell expression of RANK ligand in acute coronary syndrome: possible role in plaque destabilization," Arteriosclerosis, Thrombosis, and Vascular Biology, vol. 26, no. 4, pp. 857-863, 2006.

[36] L. Wang, X. B. Hu, W. Zhang et al., "Dickkopf-1 as a novel predictor is associated with risk stratification by GRACE risk scores for predictive value in patients with acute coronary syndrome: a retrospective research," PLoS ONE, vol. 8, no. 1, Article ID e54731, 2013.

[37] S. Lattanzio, F. Santilli, R. Liani et al., "Circulating dickkopf-1 in diabetes mellitus: association with platelet activation and effects of improved metabolic control and low-dose aspirin," Journal of the American Heart Association, vol. 3, no. 4, Article ID e001000, 2014.

[38] J. B. Cannata-Andia, P. Roman-Garcia, and K. Hruska, "The connections between vascular calcification and bone health," Nephrology Dialysis Transplantation, vol. 26, no. 11, pp. 34293436, 2011.

[39] G. Goliasch, F. Wiesbauer, S. P. Kastl et al., "Premature myocardial infarction is associated with low serum levels of Wnt-1," Atherosclerosis, vol. 222, no. 1, pp. 251-256, 2012.

[40] K.-I. Kim, K. U. Park, E. J. Chun et al., "A novel biomarker of coronary atherosclerosis: serum DKK1 concentration correlates with coronary artery calcification and atherosclerotic plaques," Journal of Korean Medical Science, vol. 26, no. 9, pp. 1178-1184, 2011.

[41] C. L. Morgan, P. Emery, D. Porter et al., "Treatment of rheumatoid arthritis with etanercept with reference to diseasemodifying anti-rheumatic drugs: long-term safety and survival using prospective, observational data," Rheumatology (United Kingdom), vol. 53, no. 1, pp. 186-194, 2014.

[42] W. G. Dixon, K. D. Watson, M. Lunt et al., "Reduction in the incidence of myocardial infarction in patients with rheumatoid arthritis who respond to anti-tumor necrosis factor $\alpha$ therapy: results from the British Society for Rheumatology Biologics Register," Arthritis and Rheumatism, vol. 56, no. 9, pp. 29052912, 2007.

[43] J. D. Greenberg, J. M. Kremer, J. R. Curtis et al., "Tumour necrosis factor antagonist use and associated risk reduction of cardiovascular events among patients with rheumatoid arthritis," Annals of the Rheumatic Diseases, vol. 70, no. 4, pp. 576-582, 2011. 


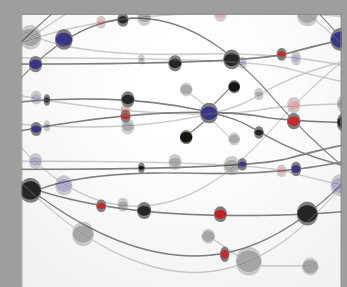

The Scientific World Journal
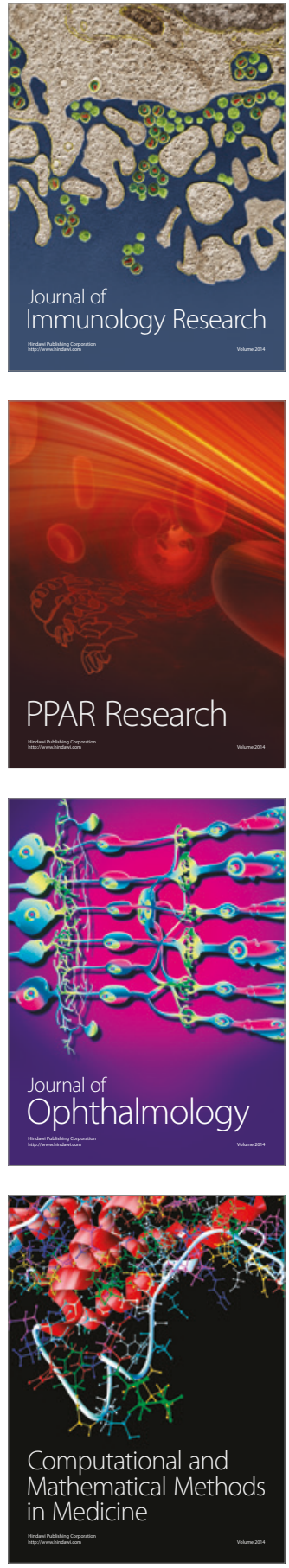

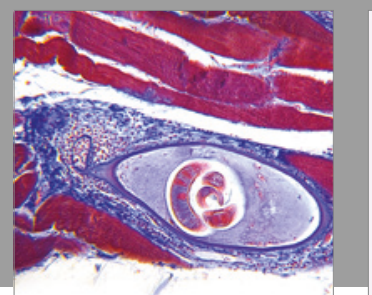

Gastroenterology Research and Practice
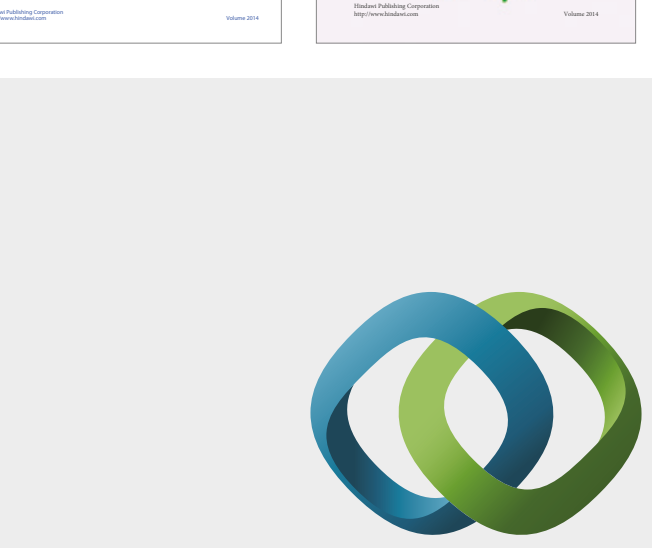

\section{Hindawi}

Submit your manuscripts at

https://www.hindawi.com
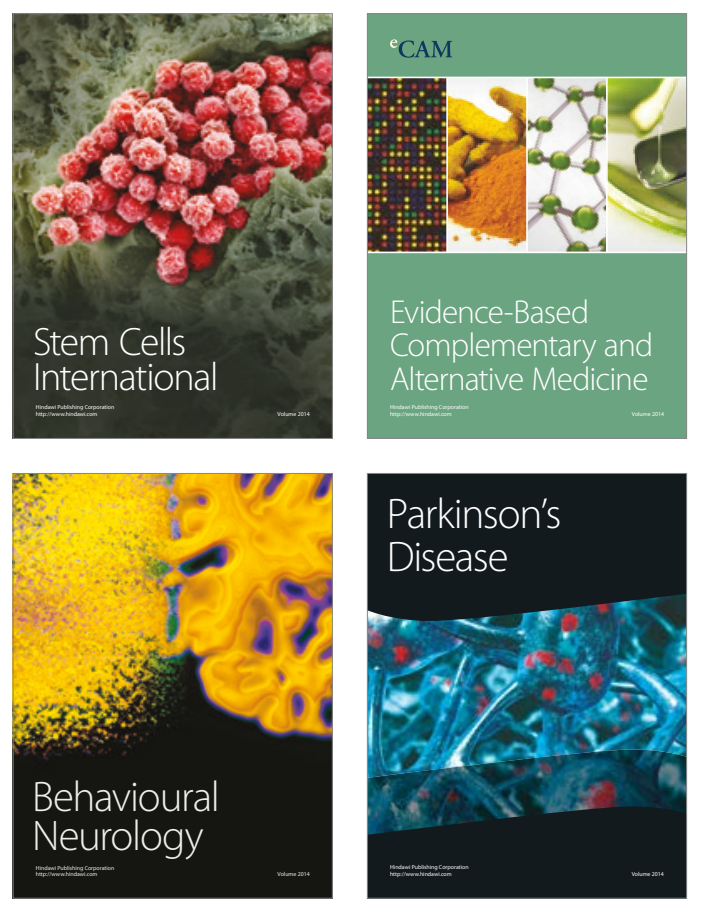
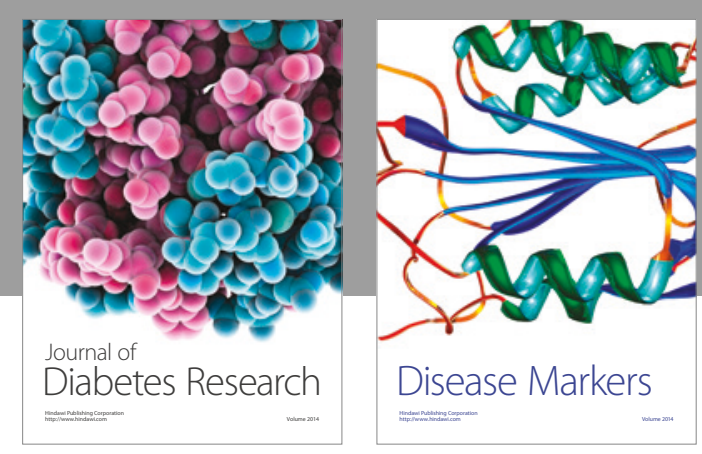

Disease Markers
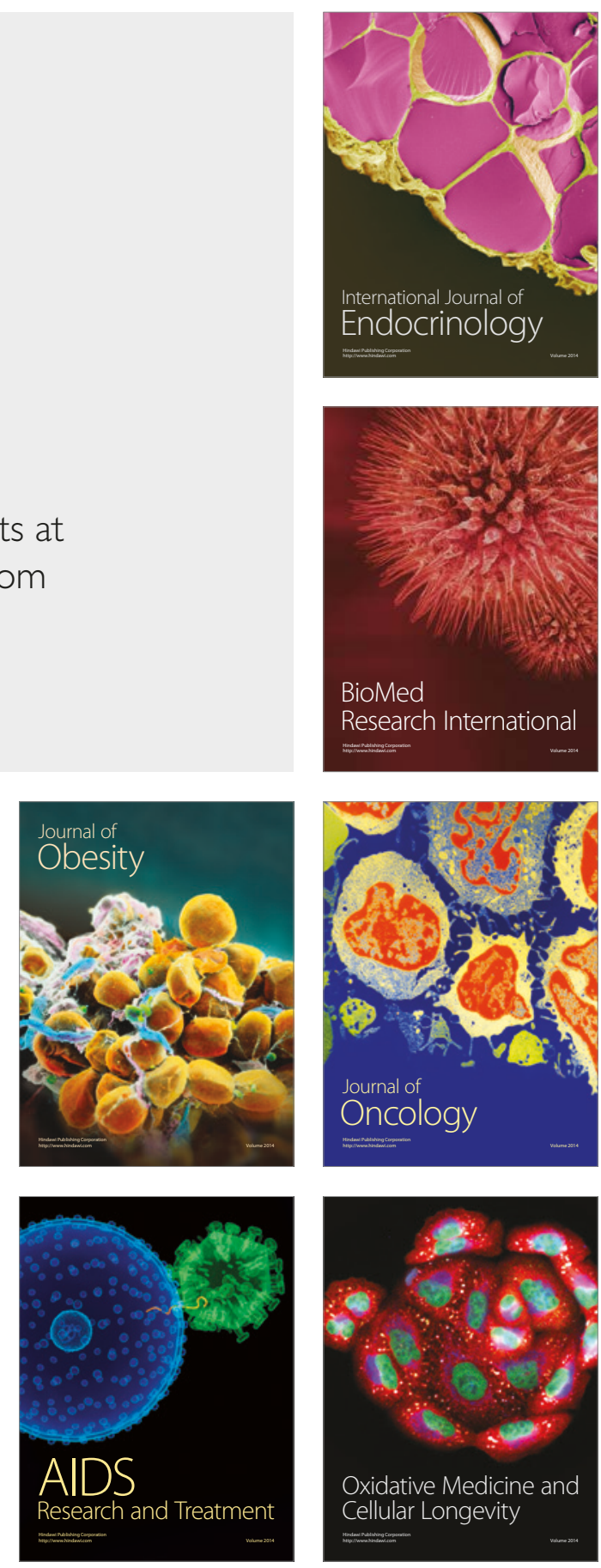